\section{Biologic DMARD May Be Insufficient to Inhibit CCL20 Pathway in Rheumatoid Arthritis}

\section{To the Editor:}

Kawashiri, et al recently reported that biologic disease-modifying antirheumatic drugs (DMARD; i.e., infliximab, etanercept, and tocilizumab) may have therapeutic efficacy by inhibiting CCL20 production in rheumatoid synovium ${ }^{1}$. Indeed, serum CCL20 concentration in patients with rheumatoid arthritis (RA) was clearly decreased by treatment with biologic DMARD. As well, DMARD also can inhibit the production of CCL20 from fibroblast-like synovial cells (FLS) in vitro ${ }^{1}$. However, the precise molecular mechanism of action remains unclear, and available evidence suggests that biologic DMARD may be of low efficiency for controlling the CCL20 pathway. For example, Kawashiri, et al also reported that CCL20 production can be significantly stimulated by interleukin 17 (IL-17) ${ }^{1}$.

Previous studies revealed that serum IL-17 levels in patients with RA did not show a significant change after infliximab and etanercept treatment $^{2,3}$. Moreover, current data have identified that gene-risk loci occur in CCR6 (a surface marker for Th17 cells and also as a receptor for CCL20 $)^{4,5}$; it is reasonable to speculate that the variant may lead to an aberrant activation of downstream signal transduction pathway. Indeed, a triallelic dinucleotide polymorphism of CCR6 (CCR6DNP) showed effects on gene transcription. The CCR6DNP genotype was correlated with the expression level of CCR6 and was associated with the presence of IL-17 in the sera of subjects with $\mathrm{RA}^{5}$. Collectively, the evidence suggests that decreased CCL20 concentration in patients with RA may be reversed by aberrant IL-17 production, and the genetic CCR6 defect may contribute to activation of a downstream pathway. Further studies are required to clarify the precise mechanism. In spite of this, data strongly indicate that biologic DMARD may be insufficient to inhibit the CCL20 pathway in patients with RA.
RUI-XUE LENG, MD; DONG-QING YE, MD, PhD, Department of Epidemiology and Biostatistics, School of Public Health, Anhui Medical University, 81 Meishan Road, Hefei, Anhui, 230032 China.

Address correspondence to Prof. D-Q. Ye; E-mail: ydq@ahmu.edu.cn

\section{REFERENCES}

1. Kawashiri SY, Kawakami A, Iwamoto N, Fujikawa K, Aramaki T, Tamai M, et al. Proinflammatory cytokines synergistically enhance the production of chemokine ligand 20 (CCL20) from rheumatoid fibroblast-like synovial cells in vitro and serum CCL20 is reduced in vivo by biologic disease-modifying antirheumatic drugs. J Rheumatol 2009;36:2397-402.

2. Kageyama Y, Kobayashi H, Kato N. Infliximab treatment reduces the serum levels of interleukin-23 in patients with rheumatoid arthritis. Mod Rheumatol 2009;19:657-62.

3. Kageyama Y, Ichikawa T, Nagafusa T, Torikai E, Shimazu M, Nagano A. Etanercept reduces the serum levels of interleukin-23 and macrophage inflammatory protein-3 alpha in patients with rheumatoid arthritis. Rheumatol Int 2007;28:137-43.

4. Stahl EA, Raychaudhuri S, Remmers EF, Xie G, Eyre S, Thomson $\mathrm{BP}$, et al. Genome-wide association study meta-analysis identifies seven new rheumatoid arthritis risk loci. Nat Genet 2010; 42:508-14.

5. Kochi Y, Okada Y, Suzuki A, Ikari K, Terao C, Takahashi A, et al. A regulatory variant in CCR6 is associated with rheumatoid arthritis susceptibility. Nat Genet 2010;42:515-9.

J Rheumatol 2010;37:11; doi:10.3899/jrheum.100551 